\title{
Partial Regularity for Nonlinear Subelliptic Systems with Dini Continuous Coefficients in Heisenberg Groups
}

\author{
Jialin Wang, Pingzhou Hong, Dongni Liao, and Zefeng Yu \\ School of Mathematics and Computer Science, Gannan Normal University, Ganzhou, Jiangxi 341000, China \\ Correspondence should be addressed to Jialin Wang; jialinwang1025@hotmail.com
}

Received 17 June 2013; Accepted 8 August 2013

Academic Editor: Pekka Koskela

Copyright (C) 2013 Jialin Wang et al. This is an open access article distributed under the Creative Commons Attribution License, which permits unrestricted use, distribution, and reproduction in any medium, provided the original work is properly cited.

\begin{abstract}
This paper is concerned with partial regularity to nonlinear subelliptic systems with Dini continuous coefficients under quadratic controllable growth conditions in the Heisenberg group $\mathbb{H}^{n}$. Based on a generalization of the technique of $\mathscr{A}$-harmonic approximation introduced by Duzaar and Steffen, partial regularity to the sub-elliptic system is established in the Heisenberg group. Our result is optimal in the sense that in the case of Hölder continuous coefficients we establish the optimal Hölder exponent for the horizontal gradients of the weak solution on its regular set.
\end{abstract}

\section{Introduction and Statements of Main Results}

In this paper, we are concerned with partial regularity of weak solutions to nonlinear sub-elliptic systems of equations of second order in the Heisenberg group $\mathbb{H}^{n}$ in divergence form, and more precisely, we consider the following systems:

$$
-\sum_{i=1}^{2 n} X_{i} A_{i}^{\alpha}(\xi, u(\xi), X u(\xi))=B^{\alpha}(\xi, u(\xi), X u(\xi)) \quad \text { in } \Omega,
$$

where $\Omega$ is a bounded domain in $\mathbb{H}^{n}, X=\left\{X_{1}, \ldots, X_{2 n}\right\}$, the definition of $X_{i}(i=1, \ldots, 2 n)$ is to be seen in the next section $(11), u=\left(u^{1}, \ldots, u^{N}\right): \Omega \rightarrow \mathbb{R}^{N}, A_{i}^{\alpha}(\xi, u, p): \mathbb{R}^{2 n+1} \times \mathbb{R}^{N} \times$ $\mathbb{R}^{2 n N} \rightarrow \mathbb{R}^{2 n N}$, and $B^{\alpha}(\xi, u, p): \mathbb{R}^{2 n+1} \times \mathbb{R}^{N} \times \mathbb{R}^{2 n N} \rightarrow \mathbb{R}^{N}$.

Under the coefficients $A_{i}^{\alpha}$ assumed to be Dini continuous, the aim of this paper is to establish optimal partial regularity to the sub-elliptic system (1) in the Heisenberg group $\mathbb{H}^{n}$. Comparing Hölder continuous coefficients (see $[1,2]$ for the case of sub-elliptic systems), such assumption is weaker. More precisely, we assume for the continuity of $A_{i}^{\alpha}$ with respect to the variables $(\xi, u)$ that

$$
\begin{gathered}
(1+|p|)^{-1}\left|A_{i}^{\alpha}(\xi, u, p)-A_{i}^{\alpha}(\tilde{\xi}, \widetilde{u}, p)\right| \\
\leq \kappa(|u|) \mu(d(\xi, \widetilde{\xi})+|u-\widetilde{u}|)
\end{gathered}
$$

for all $\xi, \tilde{\xi} \in \Omega, u, \tilde{u} \in \mathbb{R}^{N}$, and $p \in \mathbb{R}^{2 n N}$, where $\kappa:(0,+\infty) \rightarrow[1,+\infty)$ is monotone nondecreasing and $\mu:(0,+\infty) \rightarrow[0,+\infty)$ is monotone nondecreasing and concave with $\mu(0+)=0$. We also required that $r \rightarrow r^{-\gamma} \mu(r)$ be nonincreasing for some $\gamma \in(0,1)$ and that

$$
M(r)=\int_{0}^{r} \frac{\mu(\rho)}{\rho} d \rho<\infty \text { for some } r>0 .
$$

We adopt the method of $\mathscr{A}$-harmonic approximation to the case of sub-elliptic systems in the Heisenberg groups and establish the optimal partial regularity result. Roughly speaking, assume additionally to the standard hypotheses (see precisely (H1), (H2), and (H4)) that $(1+|p|)^{-1} A_{i}^{\alpha}(\xi, u, p)$ satisfies (2) and (3). Let $u \in H W^{1,2}\left(\Omega, \mathbb{R}^{N}\right)$ be a weak solution of the sub-elliptic system (1). Then, $u$ is of class $C^{1}$ outside a closed singular set Singu $\subset \Omega$ of the Haar measure 0 . Furthermore, for $\xi_{0} \in \Omega \backslash \operatorname{Sing} u$, the derivative $X u$ of $u$ has the modulus of continuity $r \rightarrow M(r)$ in a neighborhood of $\xi_{0}$. Our result is optimal in the sense that in the case $\mu(r)=r^{\gamma}$, $0<\gamma<1$, we have $M(r)=\gamma^{-1} r^{\gamma}$ Hölder continuity $\Gamma^{1, \gamma}$ to be optimal in that case.

As is well known, even under reasonable assumptions on $A_{i}^{\alpha}$ and $B^{\alpha}$ of the systems of equations, one cannot, in general, expect that weak solutions of (1) will be classical, that is, $C^{2}$-solutions. This was first shown by de Giorgi [3]; 
we also refer the reader to Giaquinta [4] and Chen and $\mathrm{Wu}$ [5] for further discussion and additional examples. Then, the goal is to establish partial regularity theory. Moreover, a new method called $\mathscr{A}$-harmonic approximation technique is introduced by Duzaar and Steffen in [6] and simplified by Duzaar and Grotowski in [7] to study elliptic systems with quadratic growth case. Then, similar results have been proved for more general $A_{i}^{\alpha}$ or $B^{\alpha}$ in the Euclidean setting; see [811] for Hölder continuous coefficients and [12-15] for Dini continuous coefficients.

However, turning to sub-elliptic equations and systems in the Heisenberg groups $\mathbb{H}^{n}$, some new difficulties will arise. Already in the first step, trying to apply the standard difference quotient method, the main difference between Euclidean $\mathbb{R}^{n}$ and Heisenberg groups $\mathbb{H}^{n}$ becomes clear. Any time we use horizontal difference quotients (i.e., in the directions $X_{i}$ ), extra terms with derivatives in the $T$ direction will arise due to noncommutativity (see (12)), but these cannot be controlled by using the initial assumptions on the weak solution. Several results were focused on those equations which have a bearing on basic vector fields on the Heisenberg group or, more generally, the Carnot group. Capogna [16, 17] studied the regularities for weak solutions to quasi-linear equations. Concretely, by a technique combining fractional difference quotients and fractional derivatives defined by Fourier transform, differentiability in the nonhorizontal direction, $W^{2,2}$ estimate, and $C^{\infty}$ continuity of weak solutions are obtained; see [16] for the case of Heisenberg groups and [17] for Carnot groups. To sub-elliptic $p$-Laplace equations in Heisenberg groups, Marchi in [18-20] showed that $T u \in L_{\text {loc }}^{p}$ and $X^{2} u \in L_{\text {loc }}^{2}$ for $1+(1 / \sqrt{5})<p<1+\sqrt{5}$ by using theories of Besov space and Bessel potential space. Domokos in $[21,22]$ improved these results for $1<p<4$ employing the A. Zygmund theory related to vector fields. Recently, by meticulous arguments, Manfredi and Mingione in [23] and Mingione et al. in [24] proved Hölder regularity with regard to full Euclidean gradient for weak solutions and further $C^{\infty}$ continuity under the coefficients assumed to be smooth.

While regularities for weak solutions to sub-elliptic systems concerning vector fields are more complicated, Capogna and Garofalo in [25] showed the partial Hölder regularity for the horizontal gradient of weak solutions to quasilinear sub-elliptic systems $-\sum_{i=1}^{k} X_{i}\left(A_{i}^{\alpha}(\xi, u) X_{j} u\right)=B^{\alpha}(\xi, u, X u)$ with $X_{i}, X_{j}(i, j=1, \ldots, k)$ being horizontal vector fields in Carnot groups of step two, where $A_{i}^{\alpha}$ and $B^{\alpha}$ satisfy the quadratic structure conditions. Their way relies mainly on generalization of classical direct method in the Euclidean setting. Shores in [26] considered a homogeneous quasilinear system $-\sum_{i=1}^{k} X_{i}\left(A_{i}^{\alpha}(\xi, u) X_{j} u\right)=0$ in the Carnot group with general step, where $A_{i}^{\alpha}$ also satisfies the quadratic growth condition. She established higher differentiability and smoothness for weak solutions of the system with constant coefficients and deduced partial regularity for weak solutions of the original system. With respect to the case of nonquadratic growth, Föglein in [27] treated the homogeneous nonlinear system $-\sum_{i=1}^{2 n} X_{i} A_{i}^{\alpha}(\xi, X u)=0$ in the Heisenberg group under superquadratic structure conditions. She got a priori estimates for weak solutions of the system with constant coefficients and partial regularity for the horizontal gradient of weak solutions to the initial system. Later, Wang and Niu [1] and Wang and Liao [2] treated more general nonlinear sub-elliptic system in the Carnot groups under superquadratic growth conditions and subquadratic growth conditions, respectively.

The regularity results for sub-elliptic systems mentioned above require Hölder continuity with respect to the coefficients $A_{i}^{\alpha}$. When the assumption of Hölder continuity on $A_{i}^{\alpha}$ is weakened to Dini continuity, how to establish partial regularity of weak solutions to nonlinear sub-elliptic systems in the Heisenberg group. This paper is devoted to this topic. To define weak solution to (1), we assume the following structure conditions on $A_{i}^{\alpha}$ and $B^{\alpha}$.

(H1) $A_{i}^{\alpha}(\xi, u, p)$ is differentiable in $p$, and there exist some constants $L$ such that

$$
\left|A_{i, p_{\beta}^{j}}^{\alpha}(\xi, u, p)\right| \leq L, \quad(\xi, u, p) \in \Omega \times \mathbb{R}^{N} \times \mathbb{R}^{2 n N} .
$$

Here, we write down $A_{i, p_{\beta}^{j}}^{\alpha}(\xi, u, p)=\left(\partial A_{i}^{\alpha}(\xi, u, p) /\right.$ $\left.\partial p_{\beta}^{j}\right)$

(H2) $A_{i}^{\alpha}(\xi, u, p)$ is uniformly elliptic; that is, for some $\lambda>$ 0 , we have

$$
A_{i, p_{\beta}^{j}}^{\alpha}(\xi, u, p) \eta_{i}^{\alpha} \eta_{j}^{\beta} \geq \lambda|\eta|^{2}, \quad \forall \eta \in \mathbb{R}^{2 n N} .
$$

(H3) There exist a modulus of continuity $\mu:(0,+\infty) \rightarrow$ $[0,+\infty)$ and a nondecreasing function $\kappa:[0,+\infty) \rightarrow$ $[1,+\infty)$ such that

$$
\begin{gathered}
(1+|p|)^{-1}\left|A_{i}^{\alpha}(\xi, u, p)-A_{i}^{\alpha}(\widetilde{\xi}, \widetilde{u}, p)\right| \\
\leq \kappa(|u|) \mu(d(\xi, \widetilde{\xi})+|u-\widetilde{u}|) .
\end{gathered}
$$

(H4) $B^{\alpha}$ satisfies quadratic controllable growth condition

$$
\left|B^{\alpha}(\xi, u, p)\right| \leq C\left(1+|u|^{r-1}+|p|^{2(1-1 / r)}\right),
$$

where $r=2 Q /(Q-2)$ because $Q>2$; see (16).

Without loss of generality, we can assume that $\kappa \geq 1$ and the following.

( $\mu 1) \mu$ is nondecreasing with $\mu(0+)=0$.

( $\mu 2) \mu$ is concave; in the proof of the regularity theorem, we have to require that $r \rightarrow r^{-\gamma} \mu(r)$ is nonincreasing for some exponent $\gamma \in(0,1)$. We also require Dini's condition (2) which was already mentioned in the introduction.

( $\mu 3) M(r)=\int_{0}^{r}(\mu(\rho) / \rho) d \rho<\infty$ for some $r>0$.

In the present paper, we will apply the method of $\mathscr{A}$ harmonic approximation adapting to the setting of Heisenberg groups to study partial regularity for the system (1). Since 
basic vector fields $X_{i}$ of Lie algebras corresponding to the Heisenberg group are more complicated than gradient vector fields in the Euclidean setting, we have to find a different auxiliary function in proving Caccioppoli type inequality. Besides, the nonhorizontal derivatives of weak solutions will happen in the Taylor type formula in the Heisenberg group and cannot be effectively controlled in the present hypotheses. So, the method employing Taylor's formula in [12] is not appropriate in our setting. In order to obtain the desired decay estimate, we use the Poincaré type inequality in [28] as a replacement. And we obtain the following main result.

Theorem 1. Assume that coefficients $A_{i}^{\alpha}$ and $B^{\alpha}$ satisfy (H1)(H4), $(\mu 1)-(\mu 3)$ and that $u \in H W^{1,2}\left(\Omega, \mathbb{R}^{N}\right)$ is a weak solution to the system (1); that is,

$$
\begin{array}{r}
\int_{\Omega} A_{i}^{\alpha}(\xi, u, X u) X_{i} \phi^{\alpha} d \xi=\int_{\Omega} B^{\alpha}(\xi, u, X u) \phi^{\alpha} d \xi \\
\forall \phi \in C_{0}^{\infty}\left(\Omega, \mathbb{R}^{N}\right) .
\end{array}
$$

Then, there exists a relatively closed set $\operatorname{Sing} u \subset \Omega$ such that $u \in C^{1}\left(\Omega \backslash \operatorname{Sing} u, \mathbb{R}^{N}\right)$. Furthermore, Sing $u \subset \Sigma_{1} \cup \Sigma_{2}$ and Haar meas $(\Omega \backslash \operatorname{Sing} u)=0$, where

$$
\begin{gathered}
\Sigma_{1}=\left\{\xi_{0} \in \Omega: \sup _{r>0}\left(\left|u_{\xi_{0}, r}\right|+\left|(X u)_{\xi_{0}, r}\right|\right)=\infty\right\}, \\
\Sigma_{2}=\left\{\xi_{0} \in \Omega: \lim _{r \rightarrow 0^{+}} \inf \left|B_{r}\left(\xi_{0}\right)\right|_{\mathbb{H}^{n}}^{-1}\right. \\
\left.\times \int_{B_{r}\left(\xi_{0}\right)}\left|X u-(X u)_{\xi_{0}, r}\right|^{2} d \xi>0\right\} .
\end{gathered}
$$

In addition, for $\tau \in[\gamma, 1)$ and $\xi_{0} \in \Omega \backslash$ Sing $u$, the derivative $X u$ has the modulus of continuity $r \rightarrow r^{\tau}+M(r)$ in a neighborhood of $\xi_{0}$.

\section{Preliminaries}

The Heisenberg group $\mathbb{H}^{n}$ is defined as $\mathbb{R}^{2 n+1}$ endowed with the following group multiplication:

$$
\begin{gathered}
\cdot: \mathbb{T}^{n} \times \mathbb{T}^{n} \longrightarrow \mathbb{H}^{n}, \\
\left(\left(\xi^{1}, t\right),\left(\widetilde{\xi}^{1}, \tilde{t}\right)\right) \longmapsto\left(\xi^{1}+\widetilde{\xi}^{1}, t+\tilde{t}+\frac{1}{2} \sum_{i=1}^{n}\left(x_{i} \tilde{y}_{i}-\widetilde{x}_{i} y_{i}\right)\right),
\end{gathered}
$$

for all $\xi=\left(\xi^{1}, t\right)=\left(x_{1}, x_{2}, \ldots, x_{n}, y_{1}, y_{2}, \ldots, y_{n}, t\right), \widetilde{\xi}=$ $\left(\widetilde{\xi}^{1}, \tilde{t}\right)=\left(\tilde{x}_{1}, \tilde{x}_{2}, \ldots, \tilde{x}_{n}, \tilde{y}_{1}, \tilde{y}_{2}, \ldots, \tilde{y}_{n}, \widetilde{t}\right)$. This multiplication corresponds to addition in Euclidean $\mathbb{R}^{2 n+1}$. Its neutral element is $(0,0)$, and its inverse to $\left(\xi^{1}, t\right)$ is given by $\left(-\xi^{1},-t\right)$. Particularly, the mapping $(\xi, \widetilde{\xi}) \mapsto \xi \cdot \widetilde{\xi}^{-1}$ is smooth, so $\left(\mathbb{H}^{n}, \cdot\right)$ is a Lie group.
The basic vector corresponding to its Lie algebra can be explicitly calculated by the exponential map and is given by

$$
X_{i}=\frac{\partial}{\partial x_{i}}-\frac{y_{i}}{2} \frac{\partial}{\partial t}, \quad X_{i+n}=\frac{\partial}{\partial y_{i}}+\frac{x_{i}}{2} \frac{\partial}{\partial t}, \quad T=\frac{\partial}{\partial t}
$$

for $i=1,2, \ldots, n$, and note that the special structure of the commutators:

$$
\begin{gathered}
{\left[X_{i}, X_{i+n}\right]=-\left[X_{i+n}, X_{i}\right]=T, \quad \text { else }\left[X_{i}, X_{j}\right]=0} \\
{[T, T]=\left[T, X_{i}\right]=0}
\end{gathered}
$$

that is, $\left(\mathbb{H}^{n}, \cdot\right)$, is a nilpotent Lie group of step $2 . X=$ $\left(X_{1}, \ldots, X_{2 n}\right)$ is called the horizontal gradient and $T$ the vertical derivative.

The pseudonorm is defined by

$$
\left\|\left(\xi^{1}, t\right)\right\|=\left(\left|\xi^{1}\right|^{4}+t^{2}\right)^{1 / 4}
$$

and the metric induced by this pseudonorm is given by

$$
d(\widetilde{\xi}, \xi)=\left\|\xi^{-1} \cdot \widetilde{\xi}\right\|
$$

The measure used on $\mathbb{\boxplus}^{n}$ is Haar measure, and the volume of the pseudoball $B_{R}\left(\xi_{0}\right)=\left\{\xi \in \mathbb{H}^{n}: d\left(\xi_{0}, \xi\right)<R\right\}$ is given by

$$
\left|B_{R}\left(\xi_{0}\right)\right|_{\mathbb{Q}^{n}}=R^{2 n+2}\left|B_{1}\left(\xi_{0}\right)\right|_{\mathbb{\square}^{n}} \triangleq \omega_{n} R^{2 n+2} .
$$

The number

$$
Q=2 n+2
$$

is called the homogeneous dimension of $\mathbb{H}^{n}$.

The horizontal Sobolev spaces $H W^{1, p}(\Omega)(1 \leq p<\infty)$ are defined as

$$
\begin{gathered}
H W^{1, p}(\Omega)=\left\{u \in L^{p}(\Omega): X_{i} u \in L^{p}(\Omega),\right. \\
i=1,2, \ldots, 2 n\} .
\end{gathered}
$$

Then, $H W^{1, p}(\Omega)$ is a Banach space with the norm

$$
\|u\|_{H W^{1, p}(\Omega)}=\|u\|_{L^{p}(\Omega)}+\sum_{i=1}^{2 n}\left\|X_{i} u\right\|_{L^{p}(\Omega)} .
$$

$H W_{0}^{1, p}(\Omega)$ is the completion of $C_{0}^{\infty}(\Omega)$ under norm (18).

Lu [28] showed the following Poincaré type inequality related to Hörmander's vector fields for $u \in H W^{1, q}\left(B_{R}\left(\xi_{0}\right)\right)$, $1<q<Q, 1 \leq p \leq q Q /(Q-q)$ :

$$
\left(\oint_{B_{R}\left(\xi_{0}\right)}\left|u-u_{\xi_{0}, R}\right|^{p} d \xi\right)^{1 / p} \leq C_{p} R\left(\oint_{B_{R}\left(\xi_{0}\right)}|X u|^{q} d \xi\right)^{1 / q}
$$

where we write down $\oint_{B_{r}\left(\xi_{0}\right)} u d \xi=\left|B_{r}\left(\xi_{0}\right)\right|_{\mathbb{T}^{n}}^{-1} \int_{B_{r}\left(\xi_{0}\right)} u d \xi$ here and there. Note the fact that the horizontal vectors $X_{i}$ defined 
in (11) fit Hörmander's vector fields and that (19) is valid for $p=q=2$.

Following [12], for technical convenience, letting $\eta(t)=$ $\mu^{2}(\sqrt{2 t})$, we have the corresponding properties for $\eta$ : $(\eta 1) \eta$ is continuous, nondecreasing and $\eta(0)=0 ;(\eta 2) \eta$ is concave, and $r \rightarrow r^{-\gamma} \eta(r)$ is nonincreasing for some exponent $\gamma \in$ $(0,1) ;(\eta 3) H(r):=4 M^{2}(\sqrt{2 r})=\left[\int_{0}^{r}(\sqrt{\eta}(\rho) / \rho) d \rho\right]^{2}<\infty$ for some $r>0$. Changing $\kappa$ by a constant, but keeping $\kappa \geq 1$, we may assume the following: $(\eta 4) \eta(1)=1$, implying $\eta(t) \geq t$ for $t \in[0,1]$. Also note that it implies that from $(\eta 2)$ and $(\eta 4)$, $\eta(t) \leq\left(\gamma^{2} / 4\right) H(t)$ for all $t \geq 0$.

Furthermore, the following inequality holds:

$$
s \eta(t) \leq s \eta(s)+t, \quad s \in[0,1], t>0 .
$$

The condition (H3) becomes

$$
\begin{aligned}
& \quad\left|A_{i}^{\alpha}(\xi, u, p)-A_{i}^{\alpha}(\tilde{\xi}, \tilde{u}, p)\right| \\
& \quad \leq \kappa(|u|) \sqrt{\eta}\left(d^{2}(\xi, \tilde{\xi})+|u-\widetilde{u}|^{2}\right)(1+|p|) .
\end{aligned}
$$

Moreover, we deduce the existence of a nonnegative modulus of continuity with $\omega(t, 0)=0$ for all $t$ such that $\omega(s, t)$ is nondecreasing with respect to $t$ for fixed $s$ and $\omega^{2}(s, t)$ is concave and nondecreasing with respect to $s$ for fixed $t$. Also, we have for $|u|+|X u| \leq M$,

$$
\begin{aligned}
& \left|A_{i, p_{\beta}^{j}}^{\alpha}(\xi, u, p)-A_{i, p_{\beta}^{j}}^{\alpha}(\tilde{\xi}, \tilde{u}, \tilde{p})\right| \\
& \quad \leq \omega\left(M, d^{2}(\xi, \widetilde{\xi})+|u-\widetilde{u}|^{2}+|p-\widetilde{p}|^{2}\right) .
\end{aligned}
$$

Using (H1) and (H2), we see that

$$
\begin{gathered}
\left|A_{i}^{\alpha}(\xi, u, p)-A_{i}^{\alpha}(\xi, u, \widetilde{p})\right| \leq L|p-\tilde{p}|, \\
\left(A_{i}^{\alpha}(\xi, u, p)-A_{i}^{\alpha}(\xi, u, \widetilde{p})\right)(p-\widetilde{p}) \geq \lambda|p-\widetilde{p}|^{2} .
\end{gathered}
$$

In the sequel, the constant $C$ may vary from line to line.

\section{Caccioppoli Type Inequality}

In this section, we present the following $\mathscr{A}$-harmonic approximation lemma in the Heisenberg group introduced by Föglein [27] with $p=2$ as a special case and prove a Caccioppoli type inequality in our setting.

Lemma 2. Let $\lambda$ and $L$ be fixed positive numbers and $n, N \in$ $\mathbb{N}$ with $n \geq 2$. If for any given $\varepsilon>0$, there exists $\delta=$ $\delta(n, N, \lambda, \varepsilon) \in(0,1]$ with the following properties:

(I) for any $\mathscr{A} \in \operatorname{Bil}\left(\mathbb{R}^{2 n N}\right)$ satisfying

$$
\mathscr{A}(\nu, \nu) \geq \lambda|\nu|^{2}, \quad \mathscr{A}(\nu, \bar{\nu}) \leq L|\nu||\bar{\nu}|, \quad \nu, \bar{\nu} \in \mathbb{R}^{2 n N}
$$

(II) for any $w \in H W^{1,2}\left(B_{\rho}\left(\xi_{0}\right), \mathbb{R}^{N}\right)$ satisfying

$$
\begin{gathered}
\oint_{B_{\rho}\left(\xi_{0}\right)}|X w|^{2} d \xi \leq 1, \\
\left|\oint_{B_{\rho}\left(\xi_{0}\right)} \mathscr{A}(X w, X \varphi) d \xi\right| \leq \delta \sup _{B_{\rho}\left(\xi_{0}\right)}|X \varphi|, \\
\forall \varphi \in C_{0}^{1}\left(B_{\rho}\left(\xi_{0}\right), \mathbb{R}^{N}\right),
\end{gathered}
$$

then, there exists an $\mathscr{A}$-harmonic function $h$ such that

$$
\oint_{B_{\rho}\left(\xi_{0}\right)}|X h|^{2} d \xi \leq 1, \quad \rho^{-2} \oint_{B_{\rho}\left(\xi_{0}\right)}|h-w|^{2} d \xi \leq \varepsilon .
$$

Föglein [27] established a priori estimate for the weak solution $u$ to homogeneous sub-elliptic systems with constant coefficients in the Heisenberg group (also see [25] for Carnot groups of step 2). We list it as follows:

$$
\sup _{B_{\rho / 2}\left(\xi_{0}\right)}\left(|u|^{2}+\rho^{2}|X u|^{2}+\rho^{4}\left|X^{2} u\right|^{2}\right) \leq C_{0} \oint_{B_{\rho}\left(\xi_{0}\right)}|X u|^{2} d \xi
$$

In what follows, we let $\rho_{1}(s, t)=(1+s+t)^{-1} \kappa(s+t)^{-1}$ and $K_{1}(s, t)=(1+t)^{4} \kappa(s+t)^{4}$ for $s, t \geq 0$. Note that $\rho_{1} \leq 1$ and that $s \rightarrow \rho_{1}(s, t), t \rightarrow \rho_{1}(s, t)$ are nonincreasing functions.

Lemma 3. Let $u \in H W^{1,2}\left(\Omega, \mathbb{R}^{N}\right)$ be a weak solution to the system (1) under the conditions (H1)-(H4), ( 1$)-(\mu 3)$. Then, for every $\xi_{0}=\left(x_{1}^{0}, x_{2}^{0}, \ldots, x_{n}^{0}, y_{1}^{0}, y_{2}^{0}, \ldots, y_{n}^{0}, t\right) \in \Omega, u_{0} \in \mathbb{R}^{N}$, $p_{0} \in \mathbb{R}^{2 n N}$, and $0<\rho<R<\rho_{1}\left(\left|u_{0}\right|,\left|p_{0}\right|\right) \leq 1$ such that $B_{R}\left(\xi_{0}\right) \subset \subset \Omega$, the inequality

$$
\begin{aligned}
& \int_{B_{\rho}\left(\xi_{0}\right)}\left|X u-p_{0}\right|^{2} d \xi \\
& \quad \leq C_{c}\left[\frac{1}{(R-\rho)^{2}} \int_{B_{R}\left(\xi_{0}\right)}\left|u-u_{0}-\left(\xi^{1}-\xi_{0}^{1}\right) p_{0}\right|^{2} d \xi+F\right]
\end{aligned}
$$

holds, where $\xi^{1}=\left(x_{1}, x_{2}, \ldots, x_{n}, y_{1}, y_{2}, \ldots, y_{n}\right)$ is the horizontal component of $\xi=\left(\xi^{1}, t\right) \in \Omega$ and

$$
\begin{aligned}
F= & \omega_{n} R^{Q} K_{1}\left(\left|u_{0}\right|,\left|p_{0}\right|\right) \eta\left(R^{2}\right) \\
& +\left[\int_{B_{R}\left(\xi_{0}\right)}\left(1+u^{r}+|X u|^{2}\right) d \xi\right]^{2(1-1 / r)} .
\end{aligned}
$$

Proof. Let $v=u-u_{0}-\left(\xi^{1}-\xi_{0}^{1}\right) p_{0}$. Take a test function $\varphi=\phi^{2} v$ in (8) with $\phi \in C_{0}^{\infty}\left(B_{R}\left(\xi_{0}\right), \mathbb{R}^{N}\right)$ satisfying $0 \leq \phi \leq 1,|\nabla \phi| \leq$ $C /(R-\rho)$, and $\phi \equiv 1$ on $B_{\rho}\left(\xi_{0}\right)$. Then, we have $X v=X u-p_{0}$, $|X \varphi| \leq \phi\left|X u-p_{0}\right|+C /(R-\rho)|v|$, and

$$
\begin{aligned}
\int_{B_{R}\left(\xi_{0}\right)} & A_{i}^{\alpha}(\xi, u, X u) \phi^{2}\left(X u-p_{0}\right) d \xi \\
= & -2 \int_{B_{R}\left(\xi_{0}\right)} \phi X \phi A_{i}^{\alpha}(\xi, u, X u) v d \xi \\
& +\int_{B_{R}\left(\xi_{0}\right)} B^{\alpha}(\xi, u, X u) \varphi^{\alpha} d \xi
\end{aligned}
$$


Adding this to the equations

$$
\begin{array}{r}
-\int_{B_{R}\left(\xi_{0}\right)} A_{i}^{\alpha}\left(\xi, u, p_{0}\right) \phi^{2}\left(X u-p_{0}\right) d \xi \\
=2 \int_{B_{R}\left(\xi_{0}\right)} \phi X \phi A_{i}^{\alpha}\left(\xi, u, p_{0}\right) v d \xi \\
-\int_{B_{R}\left(\xi_{0}\right)} A_{i}^{\alpha}\left(\xi, u, p_{0}\right) X \varphi^{\alpha} d \xi \\
0=\int_{B_{R}\left(\xi_{0}\right)} A_{i}^{\alpha}\left(\xi_{0}, u_{0}, p_{0}\right) X \varphi^{\alpha}
\end{array}
$$

It follows that by using the hypotheses (H1), (H3) (i.e., (23), (21), resp.), and (H4),

$$
\begin{aligned}
\int_{B_{R}\left(\xi_{0}\right)}\left[A_{i}^{\alpha}(\xi, u, X u)\right. \\
\left.\quad-A_{i}^{\alpha}\left(\xi, u, p_{0}\right)\right] \phi^{2}\left(X u-p_{0}\right) d \xi \\
=2 \int_{B_{R}\left(\xi_{0}\right)}\left[A_{i}^{\alpha}\left(\xi, u, p_{0}\right)\right. \\
+\int_{B_{R}\left(\xi_{0}\right)}\left[A_{i}^{\alpha}\left(\xi, u_{0}+(\xi, u, X u)\right] \phi v X \phi d \xi\right. \\
\left.\quad-A_{i}^{\alpha}\left(\xi, u, p_{0}\right)\right] X \varphi^{\alpha} d \xi \\
+\int_{B_{R}\left(\xi_{0}\right)}\left[A_{i}^{\alpha}\left(\xi, p_{0}\right)\right. \\
\quad-A_{i}^{\alpha}\left(\xi, p_{0}\right) \\
+\int_{B_{R}\left(\xi_{0}\right)} B^{\alpha}(\xi, u, X u) \varphi^{\alpha} d \xi \\
\left.\left.\left.+I I+I I+I V+V, \xi_{0}^{1}\right) p_{0}, p_{0}\right)\right] X \varphi^{\alpha} d \xi
\end{aligned}
$$

where

$$
\begin{aligned}
I= & 2 L \int_{B_{R}\left(\xi_{0}\right)}\left|X u-p_{0}\right||\phi||v||X \phi| d \xi, \\
I I= & \left(1+\left|p_{0}\right|\right) \kappa\left(\left|u_{0}\right|+R\left|p_{0}\right|\right) \\
& \times \int_{B_{R}\left(\xi_{0}\right)} \sqrt{\eta}\left(|v|^{2}\right)\left|X u-p_{0}\right| \phi^{2} d \xi,
\end{aligned}
$$

$$
\begin{gathered}
I I I=2\left(1+\left|p_{0}\right|\right) \kappa\left(\left|u_{0}\right|+R\left|p_{0}\right|\right) \\
\times \int_{B_{R}\left(\xi_{0}\right)} \sqrt{\eta}\left(|v|^{2}\right)|v||X \phi||\phi| d \xi \\
I V=\left(1+\left|p_{0}\right|\right) \kappa\left(\left|u_{0}\right|+R\left|p_{0}\right|\right) \\
\times \int_{B_{R}\left(\xi_{0}\right)} \sqrt{\eta}\left(R^{2}\left(1+\left|p_{0}\right|^{2}\right)\right)\left[\left|X u-p_{0}\right| \phi^{2}\right. \\
V=C \int_{B_{R}\left(\xi_{0}\right)}(1+|\psi||v||X \phi|] d \xi
\end{gathered}
$$

Applying (H2), the left hand side of (33) can be estimated as

$$
\begin{aligned}
& \lambda \int_{B_{R}\left(\xi_{0}\right)}\left|X u-p_{0}\right|^{2} \phi^{2} d \xi \\
& \quad \leq \int_{B_{R}\left(\xi_{0}\right)}\left[A_{i}^{\alpha}(\xi, u, X u)-A_{i}^{\alpha}\left(\xi, u, p_{0}\right)\right] \phi^{2}\left(X u-p_{0}\right) d \xi .
\end{aligned}
$$

For $\varepsilon>0$ to be fixed later, we have, using Young's inequality,

$$
I \leq \varepsilon \int_{B_{R}\left(\xi_{0}\right)}\left|X u-p_{0}\right|^{2}|\phi|^{2} d \xi+\frac{C L^{2}}{(R-\rho)^{2}} \int_{B_{R}\left(\xi_{0}\right)}|v|^{2} d \xi .
$$

Using Jensen's inequality, (20), and the fact that $\eta(t s) \leq t \eta(s)$ for $t \geq 1$, we arrive at

$$
\begin{aligned}
&\left(1+\left|p_{0}\right|\right)^{2} \kappa^{2}(\cdot) \int_{B_{R}\left(\xi_{0}\right)} \eta\left(|v|^{2}\right) d \xi \\
& \leq \omega_{n} R^{Q-2}\left(1+\left|p_{0}\right|\right)^{2} \kappa^{2}(\cdot) R^{2} \eta\left(\oint_{B_{R}\left(\xi_{0}\right)}|v|^{2} d \xi\right) \\
& \leq \omega_{n} R^{Q-2}\left[\oint_{B_{R}\left(\xi_{0}\right)}|v|^{2} d \xi\right. \\
&+\left(1+\left|p_{0}\right|\right)^{2} \kappa^{2}(\cdot) R^{2} \eta \\
&\left.\times\left(\left(1+\left|p_{0}\right|\right)^{2} \kappa^{2}(\cdot) R^{2}\right)\right]
\end{aligned}
$$

$$
\begin{aligned}
\leq & R^{-2} \int_{B_{R}\left(\xi_{0}\right)}|v|^{2} d \xi \\
& +\omega_{n} R^{Q}\left(1+\left|p_{0}\right|\right)^{4} \kappa^{4}(\cdot) \eta\left(R^{2}\right),
\end{aligned}
$$

where $\kappa(\cdot)$ is an abbreviation of the function $\kappa\left(\left|u_{0}\right|+\left|p_{0}\right|\right)$. Also, note that the application of (20) in the second last inequality is possible by our choice $R \leq \rho_{1}\left(\left|u_{0}\right|+\left|p_{0}\right|\right)$. 
Using Young's inequality and (37) in $I I$, we obtain

$$
\begin{aligned}
I I \leq & \varepsilon \int_{B_{R}\left(\xi_{0}\right)}\left|X u-p_{0}\right|^{2}|\phi|^{2} d \xi \\
& +\varepsilon^{-1}\left(1+\left|p_{0}\right|\right)^{2} \kappa^{2}(\cdot) \int_{B_{R}\left(\xi_{0}\right)} \eta\left(|v|^{2}\right) d \xi \\
\leq & \varepsilon \int_{B_{R}\left(\xi_{0}\right)}\left|X u-p_{0}\right|^{2}|\phi|^{2} d \xi \\
& +\frac{1}{\varepsilon(R-\rho)^{2}} \int_{B_{R}\left(\xi_{0}\right)}|v|^{2} d \xi \\
& +\varepsilon^{-1} \omega_{n} R^{Q}\left(1+\left|p_{0}\right|\right)^{4} \kappa^{4}(\cdot) \eta\left(R^{2}\right) .
\end{aligned}
$$

And similarly, we see

$$
\begin{aligned}
& I I I \leq \frac{4 C}{(R-\rho)^{2}} \int_{B_{R}\left(\xi_{0}\right)}|v|^{2} d \xi \\
& +\left(1+\left|p_{0}\right|\right)^{2} \kappa^{2}(\cdot) \int_{B_{R}\left(\xi_{0}\right)} \eta\left(|v|^{2}\right) d \xi \\
& \leq \frac{C}{(R-\rho)^{2}} \int_{B_{R}\left(\xi_{0}\right)}|v|^{2} d \xi \\
& +\omega_{n} R^{Q}\left(1+\left|p_{0}\right|\right)^{4} \kappa^{4}(\cdot) \eta\left(R^{2}\right), \\
& I V \leq \varepsilon \int_{B_{R}\left(\xi_{0}\right)}\left|X u-p_{0}\right|^{2}|\phi|^{2} d \xi \\
& +\frac{4 C \varepsilon}{(R-\rho)^{2}} \int_{B_{R}\left(\xi_{0}\right)}|v|^{2} d \xi \\
& +\varepsilon^{-1} \omega_{n} R^{Q}\left(1+\left|p_{0}\right|\right)^{2} \kappa^{2}(\cdot) \eta \\
& \times\left(\oint_{B_{R}\left(\xi_{0}\right)} R^{2}\left(1+\left|p_{0}\right|^{2}\right) d \xi\right) \\
& \leq \varepsilon \int_{B_{R}\left(\xi_{0}\right)}\left|X u-p_{0}\right|^{2}|\phi|^{2} d \xi \\
& +\frac{C \varepsilon}{(R-\rho)^{2}} \int_{B_{R}\left(\xi_{0}\right)}|v|^{2} d \xi \\
& +\varepsilon^{-1} \omega_{n} R^{Q}\left(1+\left|p_{0}\right|\right)^{4} \kappa^{4}(\cdot) \eta\left(R^{2}\right) .
\end{aligned}
$$

Here we have used $\kappa \geq 1$ in the last inequality.
By Hölder's inequality, (19), and Young's inequality, one gets

$$
\begin{aligned}
V \leq & C\left(\int_{B_{R}\left(\xi_{0}\right)}|\varphi|^{r} d \xi\right)^{1 / r} \\
& \times\left(\int_{B_{R}\left(\xi_{0}\right)}\left(1+|u|^{r}+|X u|^{2}\right) d \xi\right)^{(r-1) / r} \\
\leq & C\left(\int_{B_{R}\left(\xi_{0}\right)}|X \varphi|^{2} d \xi\right)^{1 / 2} \\
& \times\left(\int_{B_{R}\left(\xi_{0}\right)}\left(1+|u|^{r}+|X u|^{2}\right) d \xi\right)^{(r-1) / r} \\
\leq & \varepsilon \int_{B_{R}\left(\xi_{0}\right)}|X \varphi|^{2} d \xi+C(\varepsilon)
\end{aligned}
$$

$$
\times\left(\int_{B_{R}\left(\xi_{0}\right)}\left(1+|u|^{r}+|X u|^{2}\right) d \xi\right)^{2(r-1) / r}
$$$$
\leq \varepsilon \int_{B_{R}\left(\xi_{0}\right)}\left|X u-p_{0}\right|^{2}|\phi|^{2} d \xi
$$$$
+\frac{C \varepsilon}{(R-\rho)^{2}} \int_{B_{R}\left(\xi_{0}\right)}|\nu|^{2} d \xi
$$$$
+C(\varepsilon)\left(\int_{B_{R}\left(\xi_{0}\right)}\left(1+|u|^{r}+|X u|^{2}\right) d \xi\right)^{2(r-1) / r},
$$

where we have used the fact that $|X \varphi| \leq \phi\left|X u-p_{0}\right|+C /(R-$ $\rho)|v|$.

Applying these estimates to (37), we obtain

$$
\begin{aligned}
(\lambda-4 \varepsilon) & \int_{B_{R}\left(\xi_{0}\right)}\left|X u-p_{0}\right|^{2} \phi^{2} d \xi \\
\leq & \frac{C(L, \varepsilon)}{(R-\rho)^{2}} \int_{B_{R}\left(\xi_{0}\right)}|v|^{2} d \xi \\
& +\left(\varepsilon^{-1}+2\right) \omega_{n} R^{Q}\left(1+\left|p_{0}\right|\right)^{4} \kappa^{4}(\cdot) \eta\left(R^{2}\right) \\
& +C(\varepsilon)\left(\int_{B_{R}\left(\xi_{0}\right)}\left(1+|u|^{r}+|X u|^{2}\right) d \xi\right)^{2(r-1) / r}
\end{aligned}
$$

Choosing $\varepsilon=\lambda / 8$, we obtain the desired inequality (29).

\section{Proof of the Main Theorem}

In this section, we will complete the proof of the partial regularity results via the following lemmas. In the sequel, we always suppose that $u \in H W^{1,2}\left(\Omega, \mathbb{R}^{N}\right)$ is a weak solution to (1) with the assumptions of $(\mathrm{H} 1)-(\mathrm{H} 4)$ and $(\mu 1)-(\mu 3)$. 
Lemma 4. Let $B_{\rho}\left(\xi_{0}\right) \subset \subset \Omega$ with $\rho \leq \rho_{1}\left(\left|u_{0}\right|,\left|p_{0}\right|\right)$ and $\varphi \in$ $C_{0}^{\infty}\left(B_{\rho}\left(\xi_{0}\right), \mathbb{R}^{N}\right)$ satisfying $|\varphi| \leq \rho^{2}$ and $\sup _{B_{\rho}\left(\xi_{0}\right)}|X \varphi| \leq 1$. Then, there exists a constant $C_{1} \geq 1$ such that

$$
\begin{aligned}
\oint_{B_{\rho}\left(\xi_{0}\right)} A_{i, p_{\beta}^{j}}^{\alpha}\left(\xi_{0}, u_{0}, p_{0}\right)\left(X u-p_{0}\right) X \varphi^{\alpha} d \xi \\
\leq C_{1}\left[\Phi\left(\xi_{0}, \rho, p_{0}\right)\right. \\
\quad+\omega\left(\left|u_{0}\right|+\left|p_{0}\right|, \Phi\left(\xi_{0}, \rho, p_{0}\right)\right) \Phi^{1 / 2}\left(\xi_{0}, \rho, p_{0}\right) \\
\left.\quad+K_{1}\left(\left|u_{0}\right|,\left|p_{0}\right|\right) \sqrt{\eta}\left(\rho^{2}\right)\right] \sup _{B_{\rho}\left(\xi_{0}\right)}|X \varphi| .
\end{aligned}
$$

Proof. Using the fact that $\int_{B_{\rho}\left(\xi_{0}\right)} A_{i}^{\alpha}\left(\xi_{0}, u_{0}, p_{0}\right) X \varphi^{\alpha} d \xi=0$ and the weak form (8), we deduce

$$
\begin{aligned}
\oint_{B_{\rho}\left(\xi_{0}\right)} & {\left[\int_{0}^{1} A_{i, p_{\beta}^{j}}^{\alpha}\left(\xi_{0}, u_{0}, \theta X u+(1-\theta) p_{0}\right)\right.} \\
& \left.\times\left(X u-p_{0}\right) d \theta\right] X \varphi^{\alpha} d \xi \\
= & \oint_{B_{\rho}\left(\xi_{0}\right)}\left[A_{i}^{\alpha}\left(\xi_{0}, u_{0}, X u\right)\right. \\
= & \left.-A_{i}^{\alpha}\left(\xi_{0}, u_{0}, p_{0}\right)\right] X \varphi^{\alpha} d \xi \\
& \oint_{B_{\rho}\left(\xi_{0}\right)}\left[A_{i}^{\alpha}\left(\xi_{0}, u_{0}, X u\right)\right. \\
& -\oint_{B_{\rho}\left(\xi_{0}\right)} B^{\alpha}(\xi, u, X u) \varphi^{\alpha} d \xi .
\end{aligned}
$$

It yields

$$
\begin{gathered}
\oint_{B_{\rho}\left(\xi_{0}\right)} A_{i, p_{\beta}^{j}}^{\alpha}\left(\xi_{0}, u_{0}, p_{0}\right)\left(X u-p_{0}\right) X \varphi^{\alpha} d \xi \\
=\oint_{B_{\rho}\left(\xi_{0}\right)}\left[\int _ { 0 } ^ { 1 } \left(A_{i, p_{\beta}^{j}}^{\alpha}\left(\xi_{0}, u_{0}, p_{0}\right)\right.\right. \\
\left.\quad-A_{i, p_{\beta}^{j}}^{\alpha}\left(\xi_{0}, u_{0}, \theta X u+(1-\theta) p_{0}\right)\right) \\
\left.\quad \times\left(X u-p_{0}\right) d \theta\right] d \xi \sup _{B_{\rho}\left(\xi_{0}\right)}|X \varphi| \\
+\oint_{B_{\rho}\left(\xi_{0}\right)}\left[A_{i}^{\alpha}\left(\xi_{0}, u_{0}, X u\right)\right.
\end{gathered}
$$$$
\left.-A_{i}^{\alpha}\left(\xi, u_{0}+p_{0}\left(\xi-\xi_{0}\right), X u\right)\right] \sup _{B_{\rho}\left(\xi_{0}\right)}|X \varphi|
$$$$
+\oint_{B_{\rho}\left(\xi_{0}\right)}\left[A_{i}^{\alpha}\left(\xi, u_{0}+p_{0}\left(\xi-\xi_{0}\right), X u\right)\right.
$$$$
\left.-A_{i}^{\alpha}(\xi, u, X u)\right] \sup _{B_{\rho}\left(\xi_{0}\right)}|X \varphi|
$$$$
+\oint_{B_{\rho}\left(\xi_{0}\right)} B^{\alpha}(\xi, u, X u) \varphi^{\alpha} d \xi
$$$$
:=I^{\prime}+I I^{\prime}+I I I^{\prime}+I V^{\prime} \text {. }
$$

Using (22), Hölder's inequality, the fact that $t \rightarrow \omega^{2}(s, t)$ is concave, and Jensen's inequality, we have

$$
\begin{aligned}
I^{\prime} \leq \sup _{B_{\rho}\left(\xi_{0}\right)}|X \varphi| \oint_{B_{\rho}\left(\xi_{0}\right)} \omega\left(\left|u_{0}\right|+\left|p_{0}\right|,\left|X u-p_{0}\right|^{2}\right)\left|X u-p_{0}\right| d \xi \\
\leq \sup _{B_{\rho}\left(\xi_{0}\right)}|X \varphi|\left[\oint_{B_{\rho}\left(\xi_{0}\right)} \omega^{2}\left(\left|u_{0}\right|+\left|p_{0}\right|,\left|X u-p_{0}\right|^{2}\right) d \xi\right]^{1 / 2} \\
\quad \times\left[\oint_{B_{\rho}\left(\xi_{0}\right)}\left|X u-p_{0}\right|^{2} d \xi\right]^{1 / 2} \\
\leq \omega\left(\left|u_{0}\right|+\left|p_{0}\right|, \Phi\left(\xi_{0}, \rho, p_{0}\right)\right) \Phi^{1 / 2}\left(\xi_{0}, \rho, p_{0}\right) \sup _{B_{\rho}\left(\xi_{0}\right)}|X \varphi| .
\end{aligned}
$$

Similarly, using (21) and the fact that $\eta(t s) \leq t \eta(s)$ for $t \geq 1$, we obtain

$$
\begin{aligned}
& I I^{\prime} \leq \sup _{B_{\rho}\left(\xi_{0}\right)}|X \varphi| \kappa(\cdot) \sqrt{\eta}\left(\rho^{2}\left(1+\left|p_{0}\right|^{2}\right)\right) \\
& \quad \times \oint_{B_{\rho}\left(\xi_{0}\right)}(1+|X u|) d \xi \\
& \leq \sup _{B_{\rho}\left(\xi_{0}\right)}|X \varphi| \kappa(\cdot) \sqrt{\eta}\left(\rho^{2}\left(1+\left|p_{0}\right|\right)^{2}\right) \\
& \quad \times \oint_{B_{\rho}\left(\xi_{0}\right)}\left(1+\left|p_{0}\right|+\left|X u-p_{0}\right|\right) d \xi \\
& \leq \sup _{B_{\rho}\left(\xi_{0}\right)}|X \varphi|\left[\left(\oint_{B_{\rho}\left(\xi_{0}\right)}\left|X u-p_{0}\right|^{2} d \xi\right)\right. \\
& \quad+\kappa^{2}(\cdot)\left(1+\left|p_{0}\right|\right)^{2} \eta\left(\rho^{2}\right) \\
& \left.\quad+\kappa(\cdot)\left(1+\left|p_{0}\right|\right)^{3} \sqrt{\eta}\left(\rho^{2}\right)\right] \\
& \leq \\
& \quad\left[\Phi\left(\xi_{0}, \rho, p_{0}\right)+2 \kappa^{2}(\cdot)\left(1+\left|p_{0}\right|\right)^{3} \sqrt{\eta}\left(\rho^{2}\right)\right] \\
& \quad \times \sup _{B_{\rho}(\xi)}|X \varphi|,
\end{aligned}
$$


where we have used the fact that $\eta\left(\rho^{2}\right) \leq \sqrt{\eta}\left(\rho^{2}\right)$ which follows from the nondecreasing property of the function $\eta(t)$, $(\eta 4)$, and our assumption $\rho \leq \rho_{1} \leq 1$.

In the same way, it follows that by using (21), (37), and (19),

$$
\begin{aligned}
I I I^{\prime} \leq \sup _{B_{\rho}\left(\xi_{0}\right)}|X \varphi| \kappa(\cdot) \oint_{B_{\rho}\left(\xi_{0}\right)} \sqrt{\eta}\left(|v|^{2}\right)(1+|X u|) d \xi \\
\leq \sup _{B_{\rho}\left(\xi_{0}\right)}|X \varphi|\left[\oint_{B_{\rho}\left(\xi_{0}\right)}\left|X u-p_{0}\right|^{2} d \xi\right. \\
+\kappa^{2}(\cdot) \oint_{B_{\rho}\left(\xi_{0}\right)} \eta\left(|v|^{2}\right) d \xi \\
\left.+\kappa(\cdot)\left(1+\left|p_{0}\right|\right) \oint_{B_{\rho}\left(\xi_{0}\right)} \sqrt{\eta}\left(|v|^{2}\right) d \xi\right] \\
\leq \sup _{B_{\rho}\left(\xi_{0}\right)}|X \varphi|\left[\Phi\left(\xi_{0}, \rho, p_{0}\right)\right. \\
+2 \rho^{-2} \oint_{B_{\rho}\left(\xi_{0}\right)}|v|^{2} d \xi+\kappa^{4}(\cdot) \eta\left(\rho^{2}\right) \\
\left.+\kappa^{2}(\cdot)\left(1+\left|p_{0}\right|\right)^{2} \sqrt{\eta}\left(\rho^{2}\right)\right] \\
\leq\left[\left(1+2 C_{p}\right) \Phi\left(\xi_{0}, \rho, p_{0}\right)\right. \\
\left.+2 \kappa^{4}(\cdot)\left(1+\left|p_{0}\right|\right)^{2} \sqrt{\eta}\left(\rho^{2}\right)\right] .
\end{aligned}
$$

Using Hölder's inequality, (19), and Young's inequality, we have

$$
\begin{aligned}
I V^{\prime} \leq & C \oint_{B_{\rho}\left(\xi_{0}\right)}\left(1+|u|^{r-1}+|X u|^{2(1-1 / r)}\right)|\varphi| d \xi \\
\leq & C \oint_{B_{\rho}\left(\xi_{0}\right)}|X u|^{2(1-1 / r)}|\varphi| d \xi \\
& +C \oint_{B_{\rho}\left(\xi_{0}\right)}\left|u-u_{0}-p_{0}\left(\xi^{1}-\xi_{0}^{1}\right)\right|^{r-1}|\varphi| d \xi \\
& +C \rho^{2}\left[1+\left(\left|u_{0}\right|+\left|p_{0}\right|\right)^{r-1}\right] \\
\leq & C\left(\oint_{B_{\rho}\left(\xi_{0}\right)}|X u|^{2} d \xi\right)^{(1-1 / r)}\left(\oint_{B_{\rho}\left(\xi_{0}\right)}|\varphi|^{r} d \xi\right)^{1 / r} \\
& +C\left(\oint_{\left.B_{\rho}\left(\xi_{0}\right)^{\mid}\left|u-u_{0}-p_{0}\left(\xi^{1}-\xi_{0}^{1}\right)\right|^{r} d \xi\right)^{(1-1 / r)}}\right. \\
& \times\left(\oint_{B_{\rho}\left(\xi_{0}\right)}|\varphi|^{r} d \xi\right)^{1 / r} \\
& +C \rho^{2}\left[1+\left(\left|u_{0}\right|+\left|p_{0}\right|\right)^{r-1}\right]
\end{aligned}
$$

$$
\begin{aligned}
\leq & C\left(\oint_{B_{\rho}\left(\xi_{0}\right)}|X u|^{2} d \xi\right)^{(1-1 / r)}\left(\oint_{B_{\rho}\left(\xi_{0}\right)}|\varphi|^{r} d \xi\right)^{1 / r} \\
& +\left(\oint_{B_{\rho}\left(\xi_{0}\right)}\left|X u-p_{0}\right|^{2} d \xi\right)^{(r / 2)(1-1 / r)} \\
& \times\left(\oint_{B_{\rho}\left(\xi_{0}\right)}|\varphi|^{r} d \xi\right)^{1 / r}+C \rho^{2}\left[1+\left(\left|u_{0}\right|+\left|p_{0}\right|\right)^{r-1}\right] \\
\leq & C\|u\|_{H W^{1,2}}\left(\oint_{B_{\rho}\left(\xi_{0}\right)}\left|X u-p_{0}\right|^{2} d \xi\right)^{(1-1 / r)} \\
& \times\left(\oint_{B_{\rho}\left(\xi_{0}\right)}|\varphi|^{r} d \xi\right)^{1 / r}+C \rho\left[1+\left(\left|u_{0}\right|+\left|p_{0}\right|\right)^{r-1}\right] \\
\leq & C\|u\|_{H W^{1,2}\left(B_{\rho}\left(\xi_{0}\right)\right)}\left(\oint_{B_{\rho}\left(\xi_{0}\right)}\left|X u-p_{0}\right|^{2} d \xi\right) \\
& +C \rho^{2 r}+C \rho^{2}\left[1+\left(\left|u_{0}\right|+\left|p_{0}\right|\right)^{r-1}\right] \\
\leq & C_{2} \Phi\left(\xi_{0}, \rho, p_{0}\right)+C \rho^{2}\left(1+\left|u_{0}\right|+\left|p_{0}\right|\right)^{r-1} \\
\leq & C C_{2} \Phi\left(\xi_{0}, \rho, p_{0}\right)+C \kappa(\cdot)\left(1+\left|u_{0}\right|+\left|p_{0}\right|\right)^{2} \sqrt{\eta}\left(\rho^{2}\right),
\end{aligned}
$$

where we have used the assumption $(\eta 4)$ and the fact that $r=$ $2 Q /(Q-2)=(2 n+4) / 2 n \leq 3$ and $C_{2}=C\|u\|_{H W^{1,2}\left(B_{\rho}\left(\xi_{0}\right)\right)} \geq$ 1. Combining these estimates, we obtain the conclusion with $C_{1}=\left(1+C_{2}+2 C_{p}\right) \geq 1$.

Lemma 5. Assume that the conditions of Lemma 2 and the following smallness conditions hold:

$$
\begin{gathered}
\omega\left(\left|u_{\xi_{0}, \rho}\right|+\left|(X u)_{\xi_{0}, \rho}\right|, \Phi\left(\xi_{0}, \rho,(X u)_{\xi_{0}, \rho}\right)\right) \\
+\Phi^{1 / 2}\left(\xi_{0}, \rho,(X u)_{\xi_{0}, \rho}\right) \leq \frac{\delta}{2}, \\
C_{3} K_{1}^{2}\left(\left|u_{\xi_{0}, \rho}\right|,\left|(X u)_{\xi_{0}, \rho}\right|\right) \eta\left(\rho^{2}\right) \leq \delta^{2}
\end{gathered}
$$

with $C_{3}=8 C_{1}^{2} C_{5}$, together with

$$
\rho \leq \rho_{1}\left(1+\left|u_{\xi_{0}, \rho}\right|, 1+\left|(X u)_{\xi_{0}, \rho}\right|\right) .
$$

Then, the following growth condition holds for $\tau \in[\gamma, 1)$

$$
\begin{aligned}
\Phi\left(\xi_{0}, \theta \rho\right) \leq & \theta^{2 \tau} \Phi\left(\xi_{0}, \rho\right) \\
& +K^{*}\left(\left|u_{\xi_{0}, 2 \theta \rho}\right|,\left|(X u)_{\xi_{0}, 2 \theta \rho}\right|\right) \eta\left(\rho^{2}\right),
\end{aligned}
$$

where one abbreviates $\Phi\left(\xi_{0}, r\right)=\Phi\left(\xi_{0}, r,(X u)_{\xi_{0}, r}\right)$ and $K^{*}(s, t)=K(s, t)+(2+s+t)^{2(r-1)}$ with $K(s, t)=\left(4 \delta^{-2}+\right.$ $\left.2^{\mathrm{Q}} C_{c}\right) K_{1}^{2}(1+s, 1+t)$.

Proof. We define $w=\left[u-u_{\xi_{0}, \rho}-p_{0}\left(\xi^{1}-\xi_{0}^{1}\right)\right] \sigma^{-1}$, where

$$
\sigma=C_{1} \sqrt{\Phi\left(\xi_{0}, \rho, p_{0}\right)+4 \delta^{-2} K_{1}^{2}\left(\left|u_{0}\right|,\left|p_{0}\right|\right) \eta\left(\rho^{2}\right)} .
$$


Then, we have $X w=\sigma^{-1}\left(X u-p_{0}\right)$. Now, we consider $B_{\rho}\left(\xi_{0}\right) \subset \subset \Omega$ such that $\rho \leq \rho_{1}\left(\left|u_{0}\right|,\left|p_{0}\right|\right)$. Applying Lemma 4 on $B_{\rho}\left(\xi_{0}\right)$ to $u$, we have for any $\varphi \in C_{0}^{\infty}\left(B_{\rho}\left(\xi_{0}\right), \mathbb{R}^{N}\right)$,

$$
\begin{aligned}
& \oint_{B_{\rho}\left(\xi_{0}\right)}|X w|^{2} d \xi=\sigma^{-2} \Phi\left(\xi_{0}, \rho, p_{0}\right) \leq \frac{1}{C_{1}^{2}} \leq 1 \\
& \oint_{B_{\rho}\left(\xi_{0}\right)} A_{i, p_{\beta}^{j}}^{\alpha}\left(\xi_{0}, u_{0}, p_{0}\right) X w X \varphi d \xi \\
& \leq\left[\Phi^{1 / 2}\left(\xi_{0}, \rho, p_{0}\right)\right. \\
& \left.\quad+\omega\left(\left|u_{0}\right|+\left|p_{0}\right|, \Phi\left(\xi_{0}, \rho, p_{0}\right)\right)+\frac{\delta}{2}\right] \sup _{B_{\rho}\left(\xi_{0}\right)}|X \varphi| .
\end{aligned}
$$

In consideration of the small condition (49), we see that (54) and (55) imply conditions (26) in Lemma 2. Also note that (H1) and (H3) imply condition (25). So, there exists an $A_{i, p_{\beta}^{j}}^{\alpha}\left(\xi_{0}, u_{0}, p_{0}\right)$-harmonic function $h \in H W^{1,2}\left(B_{\rho}\left(\xi_{0}\right), \mathbb{R}^{N}\right)$ such that

$$
\oint_{B_{\rho}\left(\xi_{0}\right)}|X h|^{2} d \xi \leq 1, \quad \rho^{-2} \oint_{B_{\rho}\left(\xi_{0}\right)}|w-h|^{2} d \xi \leq \varepsilon .
$$

Taking $u_{0}=u_{\xi_{0}, 2 \theta \rho}, \theta \in(0,1 / 4]$ and replacing $p_{0}$ by $p_{0}+$ $\sigma(X h)_{\xi_{0}, 2 \theta \rho}$, we use Lemma 3 to obtain

$$
\begin{aligned}
& \int_{B_{\theta \rho}\left(\xi_{0}\right)}\left|X u-p_{0}-\sigma(X h)_{\xi_{0}, 2 \theta \rho}\right|^{2} d \xi \\
& \leq C_{c}\left[\frac{1}{(\theta \rho)^{2}} \int_{B_{2 \theta \rho}\left(\xi_{0}\right)} \mid\right. \mid-u_{\xi_{0}, 2 \theta \rho} \\
&-\left(p_{0}+\sigma(X h)_{\xi_{0}, 2 \theta \rho}\right) \\
&\left.\times\left.\left(\xi^{1}-\xi_{0}^{1}\right)\right|^{2} d \xi+F\right],
\end{aligned}
$$

where

$$
\begin{aligned}
F= & \omega_{n}(2 \theta \rho)^{Q} K_{1}\left(\left|u_{\xi_{0}, 2 \theta \rho}\right|,\left|p_{0}+\sigma(X h)_{\xi_{0}, 2 \theta \rho}\right|\right) \eta\left((2 \theta \rho)^{2}\right) \\
& +\left[\int_{B_{2 \theta \rho}\left(\xi_{0}\right)}\left(1+u^{r}+|X u|^{2}\right) d \xi\right]^{2(1-1 / r)} .
\end{aligned}
$$

Using the fact that $u-\left(p_{0}+\sigma(X h)_{\xi_{0}, 2 \theta \rho}\right)\left(\xi^{1}-\xi_{0}^{1}\right)$ has mean value $u_{\xi_{0}, 2 \theta \rho}$ on the ball $B_{2 \theta \rho}\left(\xi_{0}\right)$, the definition of $w$, and (19), we have

$$
\begin{aligned}
& \frac{1}{(\theta \rho)^{2}} \oint_{B_{2 \theta \rho}\left(\xi_{0}\right)}\left|u-u_{\xi_{0}, 2 \theta \rho}-\left(p_{0}+\sigma(X h)_{\xi_{0}, 2 \theta \rho}\right)\left(\xi^{1}-\xi_{0}^{1}\right)\right|^{2} d \xi \\
& \leq \frac{4 \sigma^{2}}{(2 \theta \rho)^{2}} \oint_{B_{2 \theta_{\rho}}\left(\xi_{0}\right)} \mid w-h_{\xi_{0}, 2 \theta \rho} \\
& \leq \frac{4 \sigma^{2}}{(2 \theta \rho)^{2}}\left[\oint_{B_{2 \theta \rho}\left(\xi_{0}\right)}|w-h|^{2} d \xi\right. \\
& \quad+\oint_{B_{2 \theta \rho}\left(\xi_{0}\right)} \mid h-h_{\xi_{0}, 2 \theta \rho} \\
& \quad-\left.(X h)_{\xi_{0}, 2 \theta \rho}\left(\xi^{1}-\xi_{0}^{1}\right)\right|^{2} d \xi \\
& \leq 4 \sigma^{2}\left[(2 \theta)^{-Q-2} \varepsilon+C_{p} \oint_{B_{2 \theta \rho}\left(\xi_{0}\right)}\left|X h-\left(\xi^{1}-\xi_{0}^{1}\right)\right|^{2} d \xi\right] \\
& \leq 4 \sigma^{2}\left[(2 \theta)^{-Q-2} \varepsilon+C_{p}^{2}(2 \theta \rho)^{2} \oint_{B_{2 \theta \rho}\left(\xi_{0}\right)}\left|X^{2} h\right|^{2} d \xi\right] \\
& \leq 4 \sigma^{2}\left[(2 \theta)^{-Q-2} \varepsilon+C_{p}^{2}(2 \theta)^{2} C_{0}\right] \\
& \leq C_{4}\left(\theta^{-Q-2} \varepsilon+\theta^{2}\right)\left[\Phi\left(\xi_{0}, \rho, p_{0}\right)\right. \\
& \left.+4 \delta^{-2} K_{1}^{2}\left(\left|u_{\xi_{0}, 2 \theta \rho}\right|,\left|p_{0}\right|\right) \eta\left(\rho^{2}\right)\right]
\end{aligned}
$$

where $C_{4}:=C_{4}(Q, \lambda, L) \geq 1$. Note that in the second last inequality we have used the fact that

$$
\begin{aligned}
\oint_{B_{2 \theta \rho}\left(\xi_{0}\right)}\left|X^{2} h\right| d \xi & \leq \sup _{B_{\rho}\left(\xi_{0}\right)}\left|X^{2} h\right| \\
& \leq C_{0} \rho^{-2} \oint_{B_{\rho}\left(\xi_{0}\right)}|X h|^{2} d \xi \leq C_{0} \rho^{-2} .
\end{aligned}
$$

In consideration of the fact that $r=2 Q /(Q-2)>2, Q \geq 4$ and the assumptions $\theta \in(0,1 / 4]$ and $\Phi \leq 1$, it follows that

$$
\begin{gathered}
{\left[\oint_{B_{2 \theta \rho}\left(\xi_{0}\right)}\left(1+u^{r}+|X u|^{2}\right) d \xi\right]^{2(1-1 / r)}} \\
\leq C\left[\oint_{B_{2 \theta \rho}\left(\xi_{0}\right)}\left|X u-p_{0}\right|^{2} d \xi\right]^{2(1-1 / r)} \\
+C\left(\oint_{B_{2 \theta \rho}\left(\xi_{0}\right)}|X u|^{2} d \xi\right)^{r-1} \\
+\left(1+\left|p_{0}\right|^{4(1-1 / r)}\right)
\end{gathered}
$$




$$
\begin{aligned}
\leq & C\left[(2 \theta)^{-2 Q(1-1 / r)} \Phi\left(\xi_{0}, \rho, p_{0}\right)^{2(1-1 / r)}\right. \\
& \left.+(2 \theta)^{-Q(r-1)} \Phi\left(\xi_{0}, \rho, p_{0}\right)^{r-1}\right] \\
& +\left(1+\left|p_{0}\right|^{2(1-1 / r)}+\left|p_{0}\right|^{4(1-1 / r)}\right) \\
\leq & C(2 \theta)^{-Q(r-1)} \Phi\left(\xi_{0}, \rho, p_{0}\right)^{2(1-1 / r)} \\
& +\left(1+\left|p_{0}\right|^{2(1-1 / r)}\right)^{2} .
\end{aligned}
$$

Let $P=p_{0}+\sigma(X h)_{\xi_{0}, 2 \theta \rho}$ with $p_{0}=(X u)_{\xi_{0}, 2 \theta \rho}$. Combining these estimates (57)-(61) and considering the small condition (51) (it implies $\rho \leq \rho_{1}\left(\left|u_{\xi_{0}, 2 \theta \rho}\right|,|P|\right)$; see (64) and (65)), we deduce that

$$
\begin{aligned}
& \Phi\left(\xi_{0}, \theta \rho\right) \leq\left|B_{\theta \rho}\left(\xi_{0}\right)\right|_{\mathbb{R}^{n}}^{-1} \int_{B_{2 \theta \rho}\left(\xi_{0}\right)}|X u-P|^{2} d \xi \\
& \leq C_{c} \frac{2^{\mathrm{Q}}}{(\theta \rho)^{2}} \oint_{B_{2 \theta \rho}\left(\xi_{0}\right)} \mid u-u_{\xi_{0}, 2 \theta \rho} \\
& -\left(p_{0}+\sigma(X h)_{\xi_{0}, 2 \theta \rho}\right) \\
& \times\left.\left(\xi^{1}-\xi_{0}^{1}\right)\right|^{2} d \xi \\
& +2^{\mathrm{Q}} C_{c} K_{1}\left(\left|u_{\xi_{0}, 2 \theta \rho}\right|,\left|p_{0}+\sigma(X h)_{\xi_{0}, 2 \theta \rho}\right|\right) \eta\left(\rho^{2}\right) \\
& +C_{c} \frac{(2 \theta \rho)^{2 Q(1-1 / r)}}{(\theta \rho)^{Q}} \\
& \times\left[\oint_{B_{2 \theta \rho}\left(\xi_{0}\right)}\left(1+u^{r}+|X u|^{2}\right) d \xi\right]^{2(1-1 / r)} \\
& \leq 2^{\mathrm{Q}} C_{4} C_{c}\left(\theta^{-\mathrm{Q}-2} \varepsilon+\theta^{2}\right) \\
& \times\left[\Phi\left(\xi_{0}, \rho\right)+4 \delta^{-2} K_{1}^{2}\right. \\
& \left.\times\left(\left|u_{\xi_{0}, 2 \theta \rho}\right|,\left|(X u)_{\xi_{0}, 2 \theta \rho}\right|\right) \eta\left(\rho^{2}\right)\right] \\
& +2^{\mathrm{Q}} C_{c} K_{1}\left(\left|u_{\xi_{0}, 2 \theta \rho}\right|, \mid(X u)_{\xi_{0}, 2 \theta \rho}\right. \\
& \left.+\sigma(X h)_{\xi_{0}, 2 \theta \rho} \mid\right) \eta\left(\rho^{2}\right) \\
& +\left[2^{\mathrm{Q}} C_{c}(2 \theta)^{2-\mathrm{Q}(r-1)} \Phi\left(\xi_{0}, \rho\right)^{2(1-1 / r)}\right. \\
& \left.+\left(1+\left|(X u)_{\xi_{0}, 2 \theta \rho}\right|^{2(1-1 / r)}\right)^{2}\right] \rho^{2}
\end{aligned}
$$

We now specify $\varepsilon=\theta^{\mathrm{Q}+4}, \theta \in(0,1 / 4]$ such that $2^{\mathrm{Q}+1} C_{4} C_{c} \theta^{2} \leq$ $\theta^{2 \tau}$. Note that the small condition (50) implies $\sigma^{2} C_{5} \leq 1$ with $C_{5}=\max \left\{C_{0}, C_{c} 2^{Q}(2 \theta)^{-\left(Q^{2}+4\right) /(Q-2)}\right\}$, and it yields

$$
\begin{aligned}
& 2^{\mathrm{Q}} C_{c}(2 \theta)^{2-Q(r-1)} \Phi\left(\xi_{0}, \rho\right)^{2(1-1 / r)} \leq 1, \\
& \left|\sigma(X h)_{\xi_{0}, 2 \theta \rho}\right| \leq \sigma \sup _{B_{2 \theta \rho}\left(\xi_{0}\right)}|X h| \\
& \leq \sigma \sqrt{C_{0}}\left(\oint_{B_{\rho}\left(\xi_{0}\right)}|X h|^{2} d \xi\right) \leq \sigma \sqrt{C_{0}} \leq 1,
\end{aligned}
$$

where we have used the a priori estimate (28) for the $\mathscr{A}$ harmonic function $h$. Furthermore, using (19) and recalling the definition of $\sigma$ and $C_{1}$, we have

$$
\begin{aligned}
\left|u_{\xi_{0}, 2 \theta \rho}\right| \leq & \left|u_{\xi_{0}, \rho}\right|+\left|u_{\xi_{0}, 2 \theta \rho}-u_{\xi_{0}, \rho}\right| \\
\leq & \left|u_{\xi_{0}, \rho}\right|+(2 \theta)^{-\mathrm{Q} / 2} \\
& \times\left(\oint_{B_{\rho}\left(\xi_{0}\right)}\left|u-(X u)_{\xi_{0}, \rho}\left(\xi^{1}-\xi_{0}^{1}\right)-u_{\xi_{0}, \rho}\right|^{2} d \xi\right)^{1 / 2} \\
\leq & \left|u_{\xi_{0}, \rho}\right|+(2 \theta)^{-\mathrm{Q} / 2} \rho \sqrt{C_{p}} \Phi^{1 / 2}\left(\xi_{0}, \rho\right) \\
\leq & \left|u_{\xi_{0}, \rho}\right|+\frac{\sigma \sqrt{C_{p}}}{C_{1}(2 \theta)^{\mathrm{Q} / 2}} \\
\leq & \left|u_{\xi_{0}, \rho}\right|+\sigma \sqrt{C_{5}} \leq\left|u_{\xi_{0}, \rho}\right|+1 .
\end{aligned}
$$

Combining these estimates with (62), we have

$$
\begin{aligned}
\Phi\left(\xi_{0}, \theta \rho\right) \leq & \theta^{2 \tau} \Phi\left(\xi_{0}, \rho\right) \\
+ & {\left[4 \delta^{-2} K_{1}^{2}\left(\left|u_{\xi_{0}, 2 \theta \rho}\right|,\left|(X u)_{\xi_{0}, 2 \theta \rho}\right|\right)\right.} \\
& +2^{Q} C_{c} K_{1}\left(\left|u_{\xi_{0}, 2 \theta \rho}\right|, \mid(X u)_{\xi_{0}, 2 \theta \rho}\right. \\
\left.\left.+\sigma(X h)_{\xi_{0}, 2 \theta \rho} \mid\right)\right] \eta\left(\rho^{2}\right) & +\left[1+\left(1+\left|(X u)_{\xi_{0}, 2 \theta \rho}\right|^{2(1-1 / r)}\right)^{2}\right] \eta\left(\rho^{2}\right) \\
\leq & \theta^{2 \tau} \Phi\left(\xi_{0}, \rho\right)+K\left(\left|u_{\xi_{0}, 2 \theta \rho}\right|,\left|(X u)_{\xi_{0}, 2 \theta \rho}\right|\right) \eta\left(\rho^{2}\right) \\
& +\left(2+\left|u_{\xi_{0}, 2 \theta \rho}\right|+\left|(X u)_{\xi_{0}, 2 \theta \rho}\right|\right)^{2(r-1)} \eta\left(\rho^{2}\right) \\
\leq & \theta^{2 \tau} \Phi\left(\xi_{0}, \rho\right)+K^{*}\left(\left|u_{\xi_{0}, 2 \theta \rho}\right|,\left|(X u)_{\xi_{0}, 2 \theta \rho}\right|\right) \eta\left(\rho^{2}\right) .
\end{aligned}
$$

Then, the proof of Lemma 5 is complete. 
For $T>0$, we find $\Phi_{0}(T)>0$ (depending on $Q, N, \lambda, L$, $\tau$, and $\omega$ ) such that

$$
\begin{gathered}
\omega^{2}\left(2 T, 2 \Phi_{0}(T)\right)+2 \Phi_{0}(T) \leq \frac{1}{2} \delta^{2}, \\
C_{1} \Phi_{0}(T) \leq \theta^{\mathrm{Q}}\left(1-\theta^{\tau}\right)^{2} T^{2} .
\end{gathered}
$$

With $\Phi_{0}(T)$ from (67), we choose $\rho_{0}(T) \in(0,1]$ (depending on $Q, N, \lambda, L, \tau, \omega, \eta$, and $\kappa$ ) such that

$$
\begin{gathered}
\rho_{0}(T) \leq \rho_{1}(1+2 T, 1+2 T), \\
C_{3} K_{1}^{2}(2 T, 2 T) \eta\left(\rho_{0}(T)^{2}\right) \leq \delta^{2}, \\
K_{0}(T) \eta\left(\rho_{0}(T)^{2}\right) \leq\left(\theta^{2 \gamma}-\theta^{2 \tau}\right) \Phi_{0}(T), \\
2\left(1+C_{p}\right) K_{0}(T) H\left(\rho_{0}(T)^{2}\right) \leq \theta^{Q}\left(1-\theta^{\gamma}\right)^{2}\left(\theta^{2 \gamma}-\theta^{2 \tau}\right) T^{2},
\end{gathered}
$$

where $K_{0}(T):=K^{*}(2 T, 2 T)$.

By the proof method of of Lemma 5.1 in [12] and conditions (67)-(68), Lemma 6 can be proved. As is well known, it is sufficient to complete the proof of Theorem 1 once we obtain Lemma 6.

Lemma 6. Assume that for some $T_{0}>0$ and $B_{\rho}\left(\xi_{0}\right) \subset \subset \Omega$ one has

$$
\begin{aligned}
& \text { (1) }\left|u_{\xi_{0}, \rho}\right|+\left|(X u)_{\xi_{0}, \rho}\right| \leq T_{0}, \\
& \text { (2) } \rho \leq \rho_{0}\left(T_{0}\right), \\
& \text { (3) } \Phi\left(\xi_{0}, \rho\right) \leq \Phi_{0}\left(T_{0}\right) .
\end{aligned}
$$

Then, the small conditions (49)-(51) are satisfied on the balls $B_{\theta^{j} \rho}\left(\xi_{0}\right)$ for $j \in N \cup\{0\}$. Moreover, the limit $\Lambda_{\xi_{0}}=$ $\lim _{j \rightarrow \infty}(X u)_{\xi_{0}, \theta^{j} \rho}$ exists, and the inequality

$$
\oint_{B_{\rho}\left(\xi_{0}\right)}\left|X u-\Lambda_{\xi_{0}}\right|^{2} d \xi \leq C_{6}\left(\left(\frac{r}{\rho}\right)^{2 \tau} \Phi\left(\xi_{0}, \rho\right)+H\left(r^{2}\right)\right)
$$

is valid for $0<r \leq \rho$ with a constant $C_{6}=C_{6}(\mathrm{Q}, N, \lambda, L$, $\tau$, and $T_{0}$ ).

Proof. The proof is very similar to the proof of Lemma 5.1 in [12]. We omit it here.

\section{Acknowledgments}

The project was supported by the National Natural Science Foundation of China (no. 11201081 and no. 11126294) and by the Science and Technology Planning Project of Jiangxi Province, China, no. GJJ13657.

\section{References}

[1] J. Wang and P. Niu, "Optimal partial regularity for weak solutions of nonlinear sub-elliptic systems in Carnot groups," Nonlinear Analysis: Theory, Methods \& Applications, vol. 72, no. 11, pp. 4162-4187, 2010.
[2] J. Wang and D. Liao, "Optimal partial regularity for subelliptic systems with sub-quadratic growth in Carnot groups," Nonlinear Analysis: Theory, Methods \& Applications, vol. 75, no. 4, pp. 2499-2519, 2012.

[3] E. de Giorgi, "Un esempio di estremali discontinue per un problema variazionale di tipo ellittico," Bollettino della Unione Matematica Italiana, vol. 4, pp. 135-137, 1968.

[4] M. Giaquinta, Multiple Integrals in the Calculus of Variations and Nonlinear Elliptic Systems, Princeton University Press, Princeton, NJ, USA, 1983.

[5] Y. Chen and L. Wu, Second Order Elliptic Equations and Elliptic Systems, Science Press, Beijing, China, 2003.

[6] F. Duzaar and K. Steffen, "Optimal interior and boundary regularity for almost minimizers to elliptic variational integrals," Journal für die Reine und Angewandte Mathematik, vol. 546, pp. 73-138, 2002.

[7] F. Duzaar and J. F. Grotowski, "Optimal interior partial regularity for nonlinear elliptic systems: the method of $A$-harmonic approximation," Manuscripta Mathematica, vol. 103, no. 3, pp. 267-298, 2000.

[8] F. Duzaar, J. F. Grotowski, and M. Kronz, "Regularity of almost minimizers of quasi-convex variational integrals with subquadratic growth," Annali di Matematica Pura ed Applicata IV, vol. 184, no. 4, pp. 421-448, 2005.

[9] F. Duzaar and G. Mingione, "The $p$-harmonic approximation and the regularity of $p$-harmonic maps," Calculus of Variations and Partial Differential Equations, vol. 20, no. 3, pp. 235-256, 2004.

[10] F. Duzaar and G. Mingione, "Regularity for degenerate elliptic problems via $p$-harmonic approximation," Annales de l'Institut Henri Poincaré. Analyse Non Linéaire, vol. 21, no. 5, pp. 735-766, 2004.

[11] S. Chen and Z. Tan, "The method of $A$-harmonic approximation and optimal interior partial regularity for nonlinear elliptic systems under the controllable growth condition," Journal of Mathematical Analysis and Applications, vol. 335, no. 1, pp. 2042, 2007.

[12] F. Duzaar and A. Gastel, "Nonlinear elliptic systems with Dini continuous coefficients," Archiv der Mathematik, vol. 78, no. 1, pp. 58-73, 2002.

[13] F. Duzaar, A. Gastel, and G. Mingione, "Elliptic systems, singular sets and Dini continuity," Communications in Partial Differential Equations, vol. 29, no. 7-8, pp. 1215-1240, 2004.

[14] Y. Qiu and Z. Tan, "Optimal interior partial regularity for nonlinear elliptic systems with Dini continuous coefficients," Acta Mathematica Scientia B, vol. 30, no. 5, pp. 1541-1554, 2010.

[15] Y. Qiu, "Optimal partial regularity of second order nonlinear elliptic systems with Dini continuous coefficients for the superquadratic case," Nonlinear Analysis: Theory, Methods \& Applications, vol. 75, no. 8, pp. 3574-3590, 2012.

[16] L. Capogna, "Regularity of quasi-linear equations in the Heisenberg group," Communications on Pure and Applied Mathematics, vol. 50, no. 9, pp. 867-889, 1997.

[17] L. Capogna, "Regularity for quasilinear equations and 1quasiconformal maps in Carnot groups," Mathematische Annalen, vol. 313, no. 2, pp. 263-295, 1999.

[18] S. Marchi, " $C^{1, \alpha}$ local regularity for the solutions of the $p$ Laplacian on the Heisenberg group for $2<p<1+\sqrt{5}$," Zeitschrift für Analysis und ihre Anwendungen, vol. 20, no. 3, pp. 617-636, 2001. 
[19] S. Marchi, " $C^{1, \alpha}$ local regularity for the solutions of the $p$ Laplacian on the Heisenberg group. The case $1+1 / \sqrt{5}<p \leq 2$," Commentationes Mathematicae Universitatis Carolinae, vol. 44, no. 1, pp. 33-56, 2003.

[20] S. Marchi, " $L^{p}$ regularity of the derivative in the second commutator direction for nonlinear elliptic equations on the Heisenberg group," Accademia Nazionale delle Scienze detta dei XL. Rendiconti. Serie V. Memorie di Matematica e Applicazioni. Parte I, vol. 26, pp. 1-15, 2002.

[21] A. Domokos, "Differentiability of solutions for the nondegenerate $p$-Laplacian in the Heisenberg group," Journal of Differential Equations, vol. 204, no. 2, pp. 439-470, 2004.

[22] A. Domokos, On the regularity of p-harmonic functions in the Heisenberg group [Ph.D. thesis], University of Pittsburgh, Pittsburgh, Pa, USA, 2004.

[23] J. J. Manfredi and G. Mingione, "Regularity results for quasilinear elliptic equations in the Heisenberg group," Mathematische Annalen, vol. 339, no. 3, pp. 485-544, 2007.

[24] G. Mingione, A. Zatorska-Goldstein, and X. Zhong, "Gradient regularity for elliptic equations in the Heisenberg group," Advances in Mathematics, vol. 222, no. 1, pp. 62-129, 2009.

[25] L. Capogna and N. Garofalo, "Regularity of minimizers of the calculus of variations in Carnot groups via hypoellipticity of systems of Hörmander type," Journal of the European Mathematical Society, vol. 5, no. 1, pp. 1-40, 2003.

[26] E. Shores, "Hypoellipticity forlinear degenerate elliptic systems in Carnot groups and applications," http://arxiv.org/abs/math/ 0502569.

[27] A. Föglein, "Partial regularity results for subelliptic systems in the Heisenberg group," Calculus of Variations and Partial Differential Equations, vol. 32, no. 1, pp. 25-51, 2008.

[28] G. Lu, "The sharp Poincaré inequality for free vector fields: an endpoint result," Revista Matemática Iberoamericana, vol. 10, no. 2, pp. 453-466, 1994. 


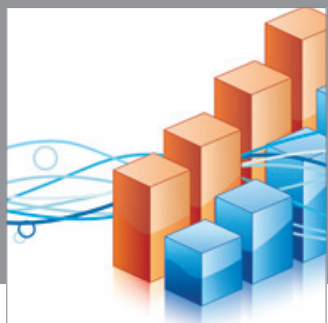

Advances in

Operations Research

mansans

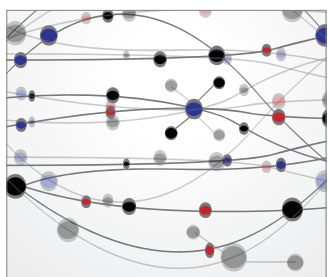

The Scientific World Journal
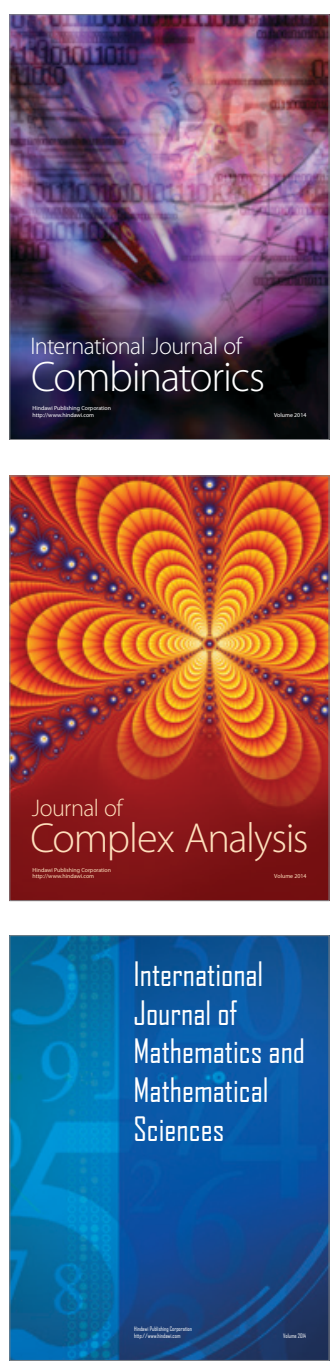
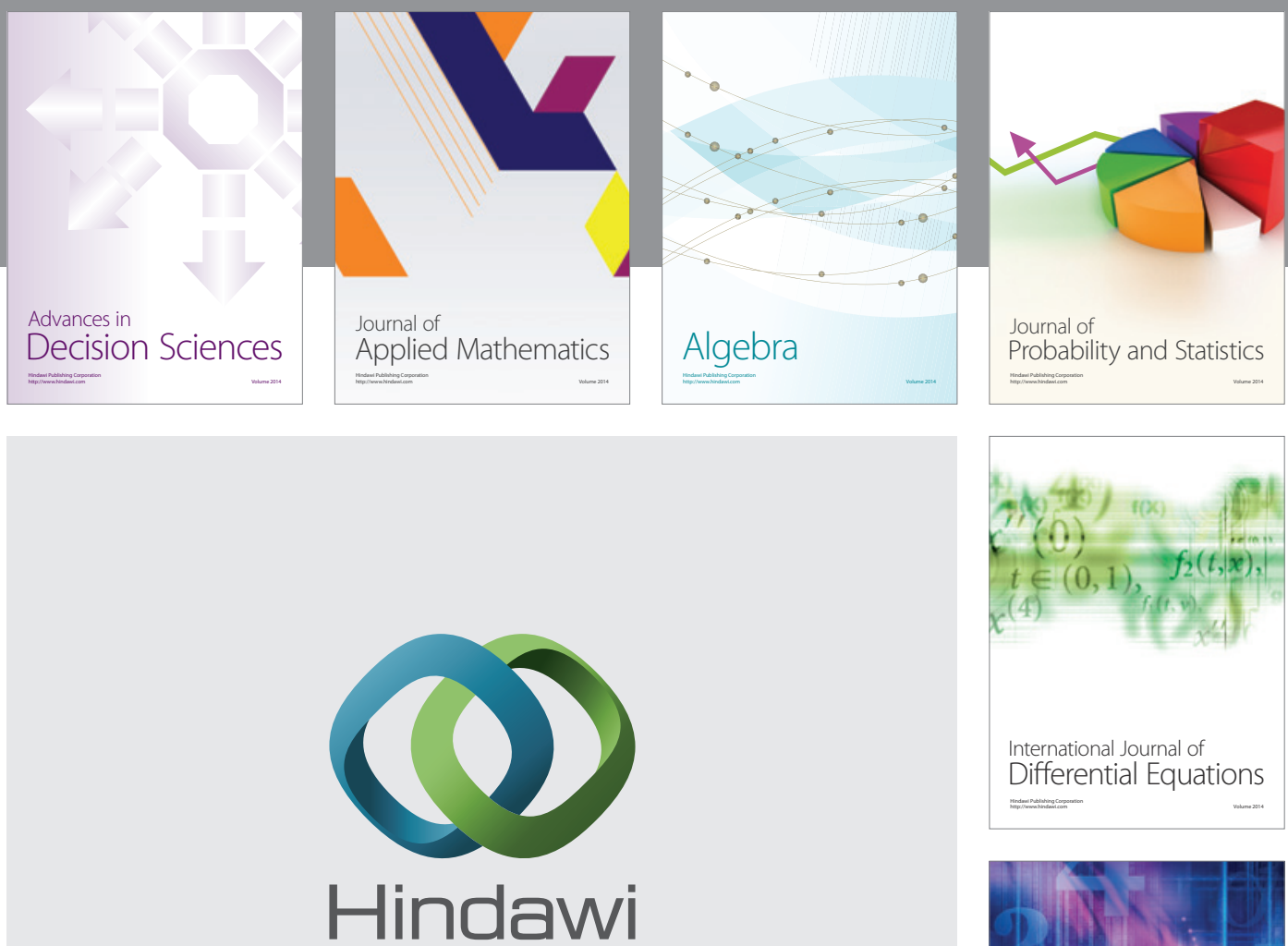

Submit your manuscripts at http://www.hindawi.com
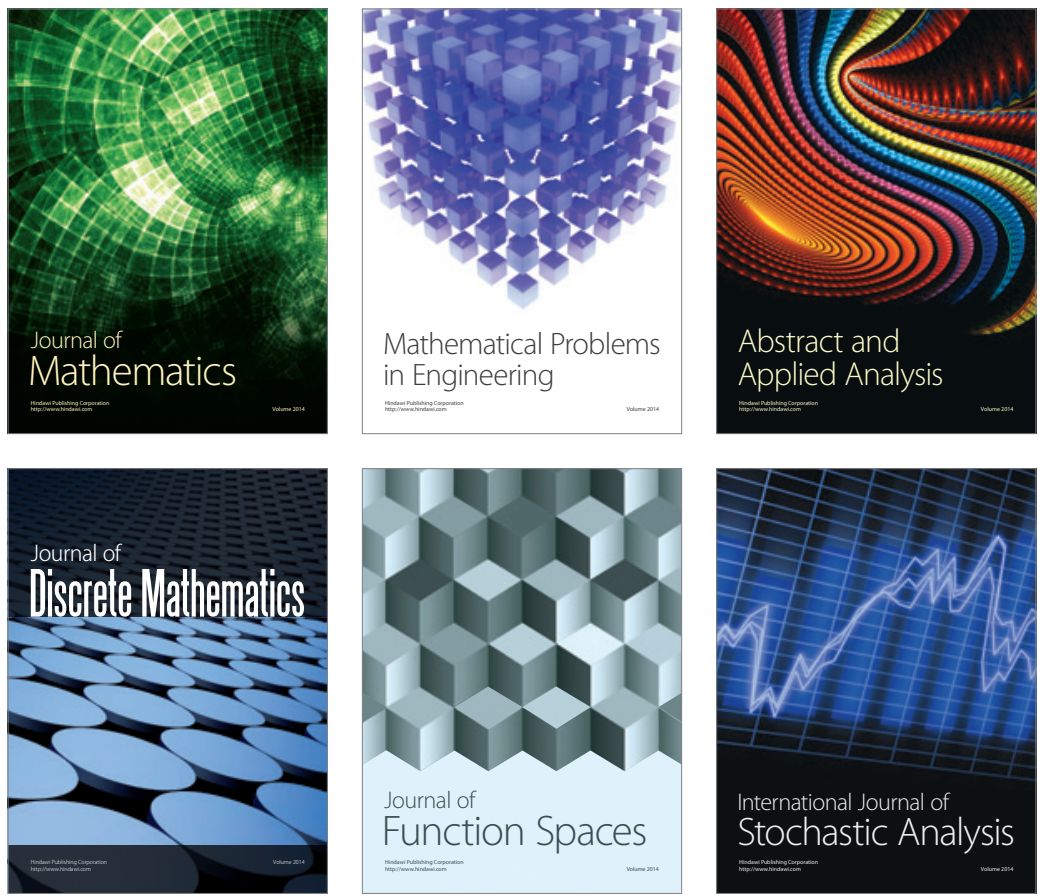

Journal of

Function Spaces

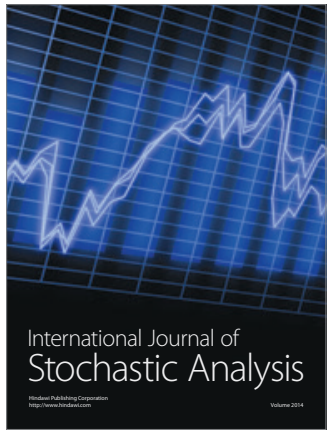

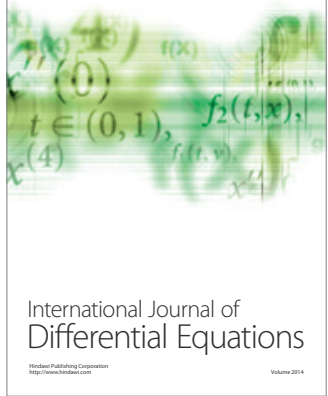
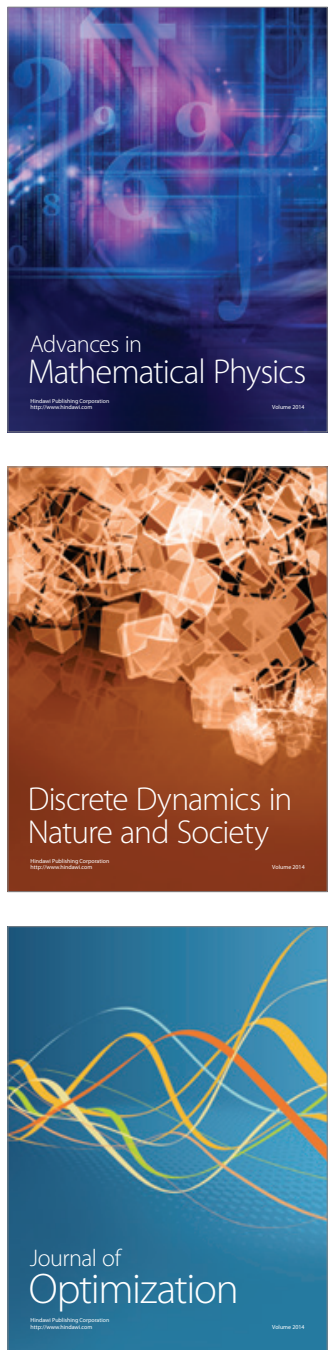